\title{
Botulinumtoxin zur Prophylaxe von Migräne und Spannungskopfschmerz
}

Vor Jahren gab es die ersten Berichte, dass Frauen, die sich wegen Falten kosmetisch mit Botulinumtoxin behandeln ließen, in der Folgezeit über eine Reduktion von vorbestehenden Kopfschmerzen (sowohl Migräne als auch Spannungskopfschmerzen) berichteten. Dies führte dann zu einer Vielzahl von zunächst offenen und später auch placebokontrollierten randomisierten Studien bei Patienten mit episodischer und chronischer Migräne, bei Patienten mit chronischen Spannungskopfschmerzen und bei Patienten mit chronischen täglichen Kopfschmerzen. Zugelassen ist Botulinumtoxin nur für die Behandlung der chronischen Migräne.

- Zehn Studien zur episodischen Migräne, sieben Studien zur chronischen Migräne, drei Studien zu chronischen täglichen Kopfschmerzen und acht Studien bei chronischen Spannungskopfschmerzen wurden jetzt einer Metaanalyse unterzogen. Endpunkte der Metaanalyse waren eine Reduktion der Kopfschmerztage pro Monat und der Anteil der Patienten mit einer mindestens 50\%igen Reduktion der Kopfschmerzhäufigkeit. Vier kleinere Studien verglichen Botulinumtoxin mit Valproinsäure, Topiramat und Amitriptylin.

Die meisten der referierten Studien waren klein und hatten in aller Regel weniger als 50 Patienten. Drei Studien zur episodischen Migräne hatten mehr als 300 Patienten. Die Metaanalyse aller Studien zur episodischen Migräne ergab 1261 Patienten die mit Botulinumtoxin A und 577, die mit Placebo behandelt wurden. Es zeigte sich kein signifikanter Unterschied der Kopfschmerzhäufigkeit pro Monat.

Bei der chronischen Migräne wurden zusammen 748 Patienten mit Botulinumtoxin und 716 mit Placebo behandelt. Hier ergab sich eine signifikante Reduktion der Kopfschmerztage verglichen mit Placebo um 2,3. Bei chro-

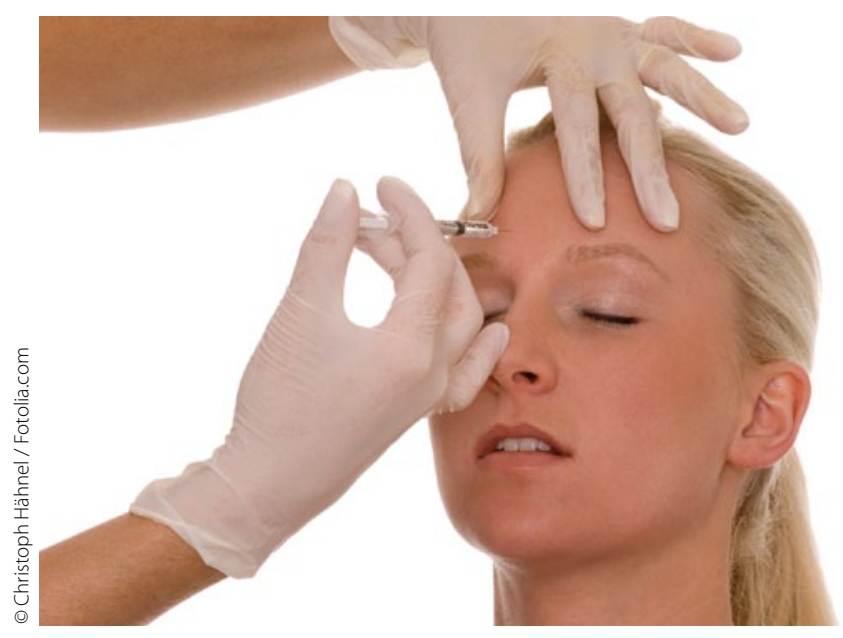

Bei chronischer Migräne wirkt Botulinumtoxin.

nischen täglichen Kopfschmerzen wurden 726 Patienten mit Botulinumtoxin behandelt und 389 mit Placebo, auch hier ergab sich ein signifikanter Unterschied zugunsten von Botulinumtoxin.

Beim chronischen Spannungskopfschmerz wurden 434 Patienten mit Botulinumtoxin behandelt und $241 \mathrm{mit}$ Placebo. Hier ergab sich eine Reduktion der Kopfschmerztage um 1,43, der Unterschied war statistisch nicht signifikant. Bezogen auf eine mindestens 50\%ige Reduktion der Kopfschmerztage waren die Patientenpopulationen so klein, dass sich daraus keine statistisch signifikanten Aussagen ableiten lassen.

Die Prophylaxe von Kopfschmerzen mit Botulinumtoxin A ist wahrscheinlich nur bei Patienten mit chronischer Migräne und bei einer Subpopulation von Patienten mit täglichen chronischen Kopfschmerzen wirksam. Der absolute Unterschied gegenüber Placebo ist gering, der absolute Therapieeffekt allerdings einschließlich des Placeboeffektes relativ hoch.

\section{Kommentar}

Botulinumtoxin ist bei der episodischen Migräne und beim chronischen Spannungskopfschmerz nicht wirksam, während es eine eindeutige Wirksamkeit bei der chronischen Migräne hat. Die Autoren der Metaanalyse kritisieren, dass der Therapieeffekt relativ gering ist, wobei sie nicht berücksichtigen, dass in der klinischen Praxis Patienten nicht mit Placebo behandelt werden und daher ein potenzieller Placeboeffekt in die Behandlungsergebnisse eingeht. Die Autoren merken zu Recht kritisch an, dass mit wenigen Ausnahmen die meisten Studien zu klein sind und keine ausreichende statis-tische Power haben. Sie kritisieren auch die häufig kurze Beobachtungszeit, wobei diese in den beiden großen Zulassungsstudien zu Botulinumtoxin bei chronischer Migräne sechs Monate betrugen mit einer weiteren sechsmonatigen Nachbeobachtungszeit. Weiterhin kommen die Autoren zu dem Schluss, dass weitere Studien zum Einsatz von Botulinumtoxin bei der Behandlung von Kopfschmerzen notwendig sind. Der Referent kann sich mit dieser Schlussfolgerung nicht anfreunden, da die Daten zur episodischen Migräne und zum chronischen Spannungskopfschmerz relativ eindeutig sind und bereits eine Zulassung für die Behandlung der chronischen Migräne vorliegt. Daher ist es sehr unwahrscheinlich, dass weitere placebokontrollierte Studien bei dieser Indikation durchgeführt werden.

H.-C. DIENER =

- J. L. Jackson, A. Kuriyama, Y. Hayashino Botulinum toxin A for prophylactic treatment of migraine and tension headaches in adults: a meta-analysis. JAMA 307 (2012) 1736-1745 